\title{
IMMUNOHISTOCHEMICAL PROFILE STRATIFIED IN AGE RANGE IN11,326 PATIENTS FROM THE BRAZILIAN UNIFIED HEALTH SYSTEM (SUS) IN SÃO PAULO
}

Catharine Coelho Santos ${ }^{1}$, Camila Santos Farabotti¹, Pedro Santos Gomes¹, Luiz Henrique Gebrim¹, André Santos Mattar $^{1}$

${ }^{1}$ Hospital Pérola Byington-São Paulo (SP), Brazil.

Introduction: The development of breast cancer is heterogeneous and there are variations in its morphologic and biological characteristics. Several risk factors for breast cancer have already been identified, including the age group. It is wellestablished that the incidence of breast cancer increases from the age of 65. Older patients are more likely to have tumors which are positive estrogen receptors (ER) and progesterone receptors (RP) and, HER2 negative, which are associated with better outcomes. In contrast, younger patients with triple-negative breast cancer and HER2 positive are associated with reduced survival and poor prognosis. Objectives: To evaluate immunohistochemical profiles stratified by age group in patients diagnosed with breast cancer at Pérola Byington Hospital. Methods: A retrospective cross-sectional study was carried out at the Referral Center for Women’s Health - Hospital Pérola Byington- São Paulo (SP), Brazil. The data were removed from the collection system during the period of January 2011 to December 2018. Such women were stratified through the subtype histochemical analysis and evaluated in relation to their age at diagnosis. Results: We analyzed the immunohistochemical profile of 11,326 patients treated at Hospital Pérola Byington from January 2011 to December 2018. Lumum tumors A corresponded to 2,629 cases and among these 7.3\% were under 40 years old, $23.1 \%$ between 40 and 50 years old and $60.5 \%$ were over 50 years old. The rate of tumors lumen B was 3,494 cases, with $10.1 \%$ in patients under $40,24.2 \%$ between 40 and 50 years old and $65.6 \%$ over 50 years old. Acheampong et al. carried out a study on the incidence of molecular subtypes in the USA between 2010 and 2016 and concluded that from the 32,0124 women included, 72.6\% were classified as luminal A, 11.2\% luminal B, $4.8 \%$ overexpression of HER2 and 11.3\% tumors. Conclusions: Based on the molecular markers, we classified the subtypes into four breast carcinomas: luminal B was the most frequent, followed by luminal A. The subtypes showed no significant difference in relation to their histological type nor age. Thus, the correlation between histological diagnosis and immunohistochemistry can improve the treatment and survival of women with breast cancer and, consequently, improve the therapeutic response of patients. 